\title{
Neuroimaging of Rapidly Progressive Dementias, Part 2: Prion, Inflammatory, Neoplastic, and Other Etiologies
}

A.J. Degnan and L.M. Levy

\begin{abstract}
SUMMARY: Most dementias begin insidiously, developing slowly and generally occurring in the elderly age group. The so-called rapidly progressive dementias constitute a different, diverse collection of conditions, many of which are reversible or treatable. For this reason, accurate identification and assessment of acute and subacute forms of dementia are critical to effective treatment; neuroimaging aids greatly in narrowing the diagnosis of these conditions. This second installment of a 2-part review of rapidly progressive dementias examines the use of imaging in an assortment of other etiologies in the differential diagnosis, from prion disease and neoplastic-related conditions to rare metabolic and other conditions such as Wernicke encephalopathy. In these clinical conditions, MR imaging has the potential to narrow this broad differential diagnosis and, at times, can definitively aid in the diagnosis of certain conditions on the basis of typical imaging patterns.
\end{abstract}

ABBREVIATIONS: CJD = Creutzfeldt-Jakob disease; $L G=$ lymphomatoid granulomatosis; SREAT = steroid-responsive encephalopathy with autoimmune thyroiditis; SSPE = subacute sclerosing panencephalitis

D ementia features prominently as a common disability among the elderly and is only expected to increase with the aging population of developed countries worldwide. Dementia constitutes diverse combinations of a loss of normal executive function in decision making, a decline in memory encoding and retrieval, and an impairment of the ability to perform cognitive tasks. In the first part of this 2-part review of rapidly progressive dementias, the neurodegenerative conditions that feature prominently in the differential diagnosis of a presenile or rapidly progressive dementia were discussed. These include the common, inexorable dementias typically associated with old age such as Alzheimer dementia and dementia with Lewy bodies. As with any clinical evaluation, imaging cannot replace accurate and thorough physical examination and history, but it contributes greatly to discerning entities within the differential diagnosis of a rapidly progressive dementia.

Rapidly progressive dementias can result from a variety of etiologies, and in this second review article, we discuss the remaining etiologies summarized in the differential diagnosis On-line Table cited in Part 1. One striking characteristic of the differential diag-

From the University of Pittsburgh Medical Center (A.J.D.), Pittsburgh, Pennsylvania; and Department of Radiology (L.M.L.), George Washington University Hospital, Washington, DC.

Please address correspondence to Lucien M. Levy, MD, 901 23rd St NW, Washington, DC 20037; e-mail: llevy@mfa.gwu.edu

-- Indicates open access to non-subscribers at www.ajnr.org

http://dx.doi.org/10.3174/ajnr.A3455

nosis for this clinical presentation of dementia is the tremendous diversity of entities encountered. This review discusses the role of neuroimaging, particularly MR imaging, in the diagnosis of a wide array of dementia-inducing conditions, including the following: prion disease such as Creutzfeldt-Jakob disease; infectious entities such as HIV-associated dementia; autoimmune and inflammatory conditions such as Hashimoto encephalopathy; malignancy-related conditions such as primary CNS lymphoma; and rare manifestations of other causes such as Wilson disease. In many of these clinical scenarios, MR imaging plays a deciding role in clarifying the clinical diagnosis. Other authors have recently reviewed, in greater detail, the clinical work-up of rapidly progressive dementias ${ }^{1,2}$; this article will focus on the consideration of these diseases when examining neuroimaging studies of rapidly progressive dementias.

\section{Prion Disease}

There are several rare disorders in which the pathophysiologic entity is the prion, a misfolded form of a normally found protein within the brain, which propagates itself by promoting changes in the conformation of the normal prion protein. The most common set of conditions involving the prion is Creutzfeldt-Jakob disease, which is either inherited in a familial fashion, sporadically occurring, or acquired through exposure. CJD may fall under both the neurodegenerative and infectious classifications; the latter is thought to occur in variant CJD or new-variant CJD, related to ingestion of mad-cow-infected beef. ${ }^{3}$ Of these types, the spo- 


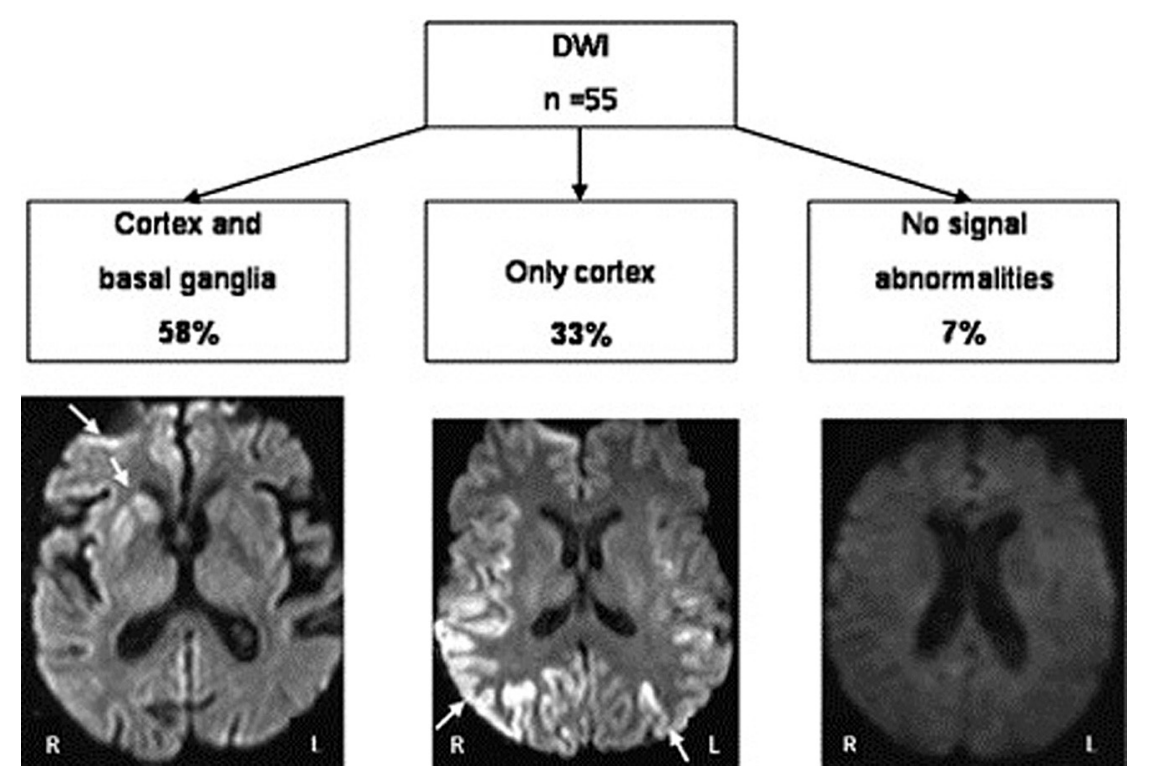

FIG 1. DWI in CJD. The most frequent MR imaging lesion patterns were defined by using DWI as the most sensitive technique. Cortex and basal ganglia hyperintensity was observed in approximately two-thirds (58\%), and isolated cortical hyperintensity, in one-third (33\%). ${ }^{7}$
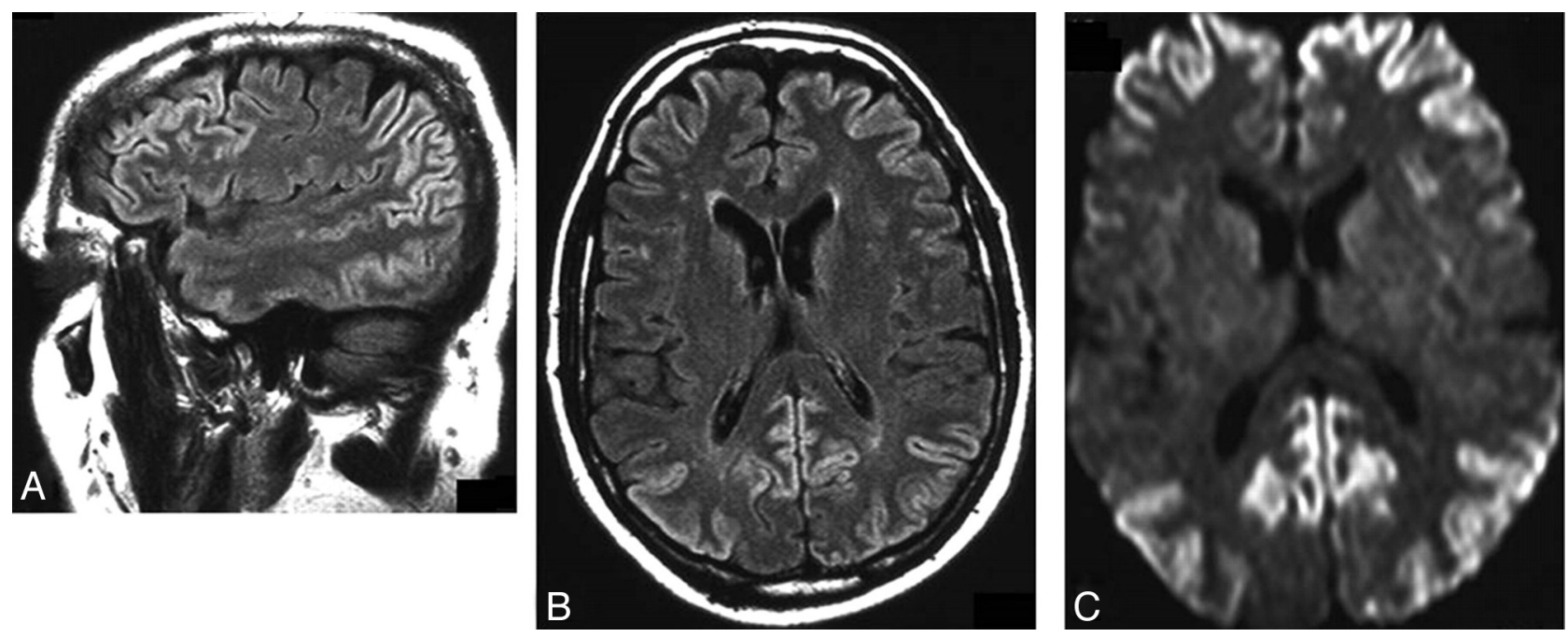

FIG 2. DWI in CJD. Involvement of the frontal and parietal lobes in patients with isolated cortical hyperintensities in the setting of CreutzfeldtJakob Disease. A, Sagittal FLAIR-weighted scan of a 58-year-old patient 3.5 months after the disease onset and 1 week before death shows signal-intensity increases in the frontal and parietal lobes. B. Axial FLAIR-weighted scan of the same patient shows frontal and parietal signal-intensity increases. C, DWI of the same patient shows frontal and parietal signal-intensity increases.

radic form is the most common, followed by genetic and acquired forms. ${ }^{2}$ This condition may differ clinically from many other conditions in the differential on the basis of its cerebellar dysfunction and common presence of myoclonus. Nevertheless, its clinical overlap with corticobasal degeneration and progressive supranuclear palsy necessitates the use of imaging to differentiate it from other rapidly progressive dementias.

The imaging findings typical of CJD, while observed on T2weighted and FLAIR sequences, are best seen by using high-bvalue DWI and are confirmed with reciprocal changes on ADC images. ${ }^{4-6}$ DWI typically demonstrates hyperintensities within the basal ganglia, the thalamus, and cortex (Figs 1 and 2). ${ }^{7}$ The "hockey stick" or "pulvinar" sign, which is formed by the confluent hyperintensity within the dorsomedial thalamus and the posterior thalamus (pulvinar), is thought to be indicative of vari- ant (infectious acquired) CJD, though these signs may be seen in other forms of CJD. ${ }^{8,9}$ These imaging changes are much more sensitive and specific than previous noninvasive clinical methods for CJD diagnosis, including the presence of CSF protein 14-3-3 and periodic sharp waves on electroencephalography or neuronspecific enolase. ${ }^{3,9}$ For this reason, imaging findings are essential in making the diagnosis of CJD, despite their not being included in earlier clinical criteria. ${ }^{10}$

\section{Infection-Related Etiologies}

In younger patients with dementia symptoms, both infectious and inflammatory-related conditions are much more frequent than the neurodegenerative diseases. Encephalitis and other infections of the CNS are generally hyperacute in onset; when cognitive decline is present, these cognitive symptoms are likewise 
hyperacute in onset. In addition, encephalopathic symptoms with marked confusion and neuropsychiatric manifestations also point clinically to this diagnosis, as do non-neurologic manifestations of infection such as cutaneous or respiratory presentations. Readers are referred to the work of Almeida and Lautenschlager $^{11}$ for a thorough clinical discussion of dementia associated with infections.

Encephalitis. The diagnosis of encephalitis in the acute setting may prove problematic: Clinical symptoms are generally nonspecific and may involve a variety of neurologic symptoms or none at all. While rarely presenting with dementia acutely, rapidly progressive dementia in younger individuals merits consideration of encephalitis. Even rapid polymerase chain reaction laboratory tests of herpes simplex may have negative findings in the very early stages of encephalitis, which make ancillary work-up imperative.

MR imaging can reveal pathologic changes manifest as FLAIR and T2 hyperintensity within the temporal (particularly, medial), insular, and inferior frontal cortices in patients with herpes encephalitis. ${ }^{12,13}$ In the much less common Japanese encephalitis, these abnormalities tend to be seen in subcortical gray matter regions. ${ }^{13}$ Patchy enhancement with gadolinium administration indicates nonspecific inflammatory changes generally seen in encephalitis. DWI augments the evaluation of encephalitis with earlier detection of changes than on conventional imaging, though it may not be as sensitive later in the clinical course. ${ }^{13-16}$ Restricted diffusion occurs in herpes encephalitis during the acute phase and appears less often in Japanese encephalitis, though other authors demonstrated diffusion changes in the thalamus in this condition. ${ }^{16,17}$ Moreover, magnetic-susceptibility-sensitive sequences (eg, gradient recalled-echo, susceptibility-weighted, and susceptibility angiography) may detect focal hemorrhage associated with sequelae of CNS infection.

\section{HIV and AIDS-Related Dementias}

The occurrence of dementia in HIV-infected individuals ranges from 1 in 10 to 1 in 3 . HIV dementia may be considered a subcortical dementia with cognitive slowing, motor retardation, and impaired memory. ${ }^{11}$ Fortunately, successful treatment with antiretroviral therapy has the potential to ameliorate cognitive symptoms and sometimes reverse cognitive deficits in HIV dementia. ${ }^{1}$ For this reason, identification and establishment of an appropriate treatment regimen is of paramount importance. Imaging is also vital to ensure that no other AIDS-related CNS conditions are present, such as primary CNS lymphoma, progressive multifocal leukoencephalopathy (seen as demyelinating white matter lesions on MR imaging), or toxoplasmosis (with multiple deep gray matter lesions). ${ }^{18}$ With cognitive decline in the setting of HIV infection, volumetric studies observed atrophy by using MR imaging. ${ }^{19}$ These changes are not generally apparent on conventional MR imaging on an individual basis. DTI may, however, show reduced fractional anisotropy inversely correlated with viral loads. ${ }^{19-21}$ Magnetization transfer ratios also appear different when comparing HIV-infected patients to controls. ${ }^{19}$

MR spectroscopy can also detect changes that reflect dementia symptoms in HIV-infected patients, regardless of neuroanatomic changes; a recent study by Ernst et $\mathrm{al}^{22}$ demonstrated reductions in parietal gray matter glutamate in subjects with HIV with cog- nitive deficits. Others have implicated decreased concentrations of NAA and combined glutamate and glutamine in HIV-infected patients with reductions in cortical, hippocampal, and subcortical volume, respectively. ${ }^{4}$

Neuroborreliosis. CNS manifestations related to Lyme disease caused by Borrelia burgdorferi are rare; contentious; and largely related to cranial nerve involvement, polyradiculopathy, or neuropsychiatric manifestations. Acute dementia in this setting has been previously reported, and though infrequent, may place this condition on the differential list of rapid-onset dementias. ${ }^{23} \mathrm{An}$ extensive review by Hildenbrand et $\mathrm{al}^{24}$ discussed the neuroimaging findings and the clinical syndrome of neuroborreliosis in greater detail. Rarely, encephalomyelitis may develop from spirochete infiltration into the brain, as indicated by tumefactive white matter lesions. ${ }^{25}$ Traditionally, more commonly seen in Lyme disease with CNS involvement are nonspecific FLAIR abnormalities predominantly within the frontal cortex; on the other hand, more recent, systematic investigation suggests that this is an uncommon occurrence with only $10 \%$ of patients with neuroborreliosis demonstrating T2 hyperintense foci. ${ }^{26,27}$ These lesions may mimic a demyelinating process; thus, in cases in which multiple sclerosis is considered a reasonable alternate explanation for T2 foci, DTI and magnetization transfer imaging are helpful in demonstrating the absence of occult white matter injury in Lyme disease in juxtaposition to multiple sclerosis. ${ }^{28}$

Other Infectious Dementias. Of importance but rarely rapid in progression is tertiary syphilis, which should be clinically determined by using appropriate laboratory testing with rapid plasma reagin testing performed in all suspected patients with rapid or presenile dementia. Cognitive decline is the most common neurologic symptom in tertiary syphilis. Early MR imaging reports describe focal hyperintensities on T2-weighted images in an arterial distribution, in keeping with meningovascular pathophysiology. ${ }^{29}$ Multiple areas of ischemia and infarction are thought to result from endarteritis in some cases of fulminant neurosyphilis. ${ }^{18}$ Cerebral gummatous disease may also occur in neurosyphilis, with lesions that are hypointense on T1- and hyperintense on T2-weighted images. ${ }^{29}$

Other infectious etiologies such as fungal encephalitides may present with dementia, but as with most infections of the central nervous system, they also tend to present with neurologic dysfunction and other clinical features more often than simple dementia. There are reports of Cryptococcus neoformans as a causative agent of rapidly progressive cognitive dysfunction, with 1 case even misdiagnosed as Alzheimer disease. ${ }^{30}$ Neurocysticercosis is rare in the developed world, but in places where it is endemic, it may present with dementia in a small fraction of cases. ${ }^{11}$

Another infectious form of sudden dementia, typically seen in a much younger population of children and adolescents (in comparison with the remainder of conditions discussed in this article) is subacute sclerosing panencephalitis, which is a rare condition caused by the measles paramyxovirus, occurring months to years after infection. MR imaging findings are generally normal early in SSPE and may progress to involve high T2 signal within white matter or hyperintense lesions in the parietal and temporal lobes in the acute stage and then progress to involve atrophy and deep 
and cortical gray matter changes. ${ }^{31-33}$ MR spectroscopy in later stages of SSPE reveals decreased NAA, consistent with neuronal loss and elevated choline, lipid, and lactate concentrations consistent with inflammation. ${ }^{34}$ More recent work indicates the use of DTI; notably, DTI detects reductions in fractional anisotropy even within normal-appearing white matter on conventional imaging, suggesting subtle disruption of white matter tracts in the early stages of SSPE. ${ }^{35}$

\section{Inflammatory and Autoimmune Conditions}

Inflammatory and autoimmune conditions are often associated with diverse clinical symptoms and an amorphous presentation. They constitute the second largest group of rapidly progressive dementias in 2 large cohorts but are a much smaller portion of another group of presenile rapid dementias. ${ }^{3,36}$

Multiple Sclerosis. Nearly 1 in 20 patients with MS manifest dementia, but the prevalence of other cognitive disturbances is much more common and subtle. ${ }^{37}$ Most imaging reports of this phenomenon attribute the cognitive decline to the presence of nonspecific neuroanatomic atrophy, without particular observation of an affected structure. ${ }^{38}$ The reason for this generalized atrophy has been discussed and examined at great length- even in normal-appearing white matter in MS, there are detectable correlates of neuronal loss with decreased NAA on MR spectroscopy within both normal white and gray matter in patients with MS. ${ }^{39}$ Moreover, these measures of atrophy in patients with MS correlate with the progression of disability and were reviewed at great length by Anderson et al. ${ }^{39}$ Nevertheless, dementia in the setting of patients with known MS should be evaluated as with any other rapidly progressive dementia, with MS-related dementia as a diagnosis of exclusion.

Hashimoto Encephalopathy. This condition related to Hashimoto thyroiditis is also referred to as steroid-responsive encephalopathy with autoimmune thyroiditis, which explains the nature of this syndrome that is believed to be related to a cross-reaction between autoimmune antithyroid antibodies and neurons, resulting in generalized CNS inflammation and neurologic impairment. ${ }^{3,40}$ Most interesting, patients may not demonstrate overt thyroid dysfunction at the time of neurologic presentation. MR imaging findings, as with many of these autoimmune conditions, are frequently normal (60\% of cases) or nonspecific in nature, involving FLAIR and T2 abnormalities within subcortical white matter and contrast enhancement of the meninges. ${ }^{40,41}$ Making the diagnosis of SREAT is important because early initiation of corticosteroid pharmacotherapy yields symptom resolution and reversal of imaging findings. ${ }^{41}$

Neurosarcoidosis. As a systemic granulomatous disease, sarcoidosis may simulate many neurologic disorders, and likewise, imaging of neurosarcoidosis is complex. ${ }^{42}$ Neurologic involvement is also relatively common in neurosarcoidosis, with approximately 1 in 4 patients with sarcoidosis demonstrating CNS involvement at postmortem examination. ${ }^{43}$ When present, the most frequent abnormalities observed on MR imaging are periventricular T2weighted hyperintense lesions, enhancing granulomas, and leptomeningeal enhancement similar to that seen in meningitis. ${ }^{42,44}$ Dural masses may be seen, which are T2 hypointense; contrast enhancement may be mistaken for meningioma, without a clinical history of sarcoidosis. ${ }^{43}$

Vasculitides. Primary CNS vasculitis is a rare entity, but systemic vasculitides may also present with CNS manifestations with cognitive deficits in half of patients. ${ }^{45,46}$ Vasculitides can be differentiated from many other dementias in that there are frequently concomitant cerebrovascular events seen on MR imaging, with hemorrhage and infarct more likely present. ${ }^{3}$ FLAIR and T2weighted hyperintensities are also prevalent in vasculitis, thickening of the vessels and extramural enhancement are more definitive findings, and findings of gadolinium enhancement within the meninges are not as commonplace as generally thought on the basis of the results of a large observational study. ${ }^{45}$

Other Inflammatory and Autoimmune Conditions. Celiac disease, an intolerance of food products containing gluten, often with the presence of autoantibodies to the enzyme tissue transglutaminase, may present with neurologic symptoms, including dementia, though those reported are quite varied, ranging from chronic headaches to ataxia. ${ }^{47,48}$ Oddly, these symptoms can occur without gastrointestinal involvement and may improve with dietary changes. ${ }^{49} \mathrm{MR}$ imaging of the brain may reveal hyperintense T2-weighted posterior white matter foci with a reported prevalence of 1 in 5 in patients with celiac disease in 1 study, independent of the presence of neurologic symptoms. ${ }^{3,50}$ Most interesting, measured cerebral perfusion on SPECT imaging in 1 small study was significantly lower within several brain regions in patients with untreated celiac disease, while there was no significant difference between patients adhering to dietary restriction and controls. ${ }^{51}$ These findings suggest a process by which patients may sustain hypoperfusion with resultant brain injury, generating lesions visible on conventional MR imaging. Nevertheless, the evidence for and mechanism of neurologic involvement and the importance of MR imaging findings in celiac disease are controversial topics without conclusions.

\section{Malignancy-Related Conditions}

Paraneoplastic CNS Involvement. There are a number of paraneoplastic neurologic disorders with a diverse set of clinical presentations that may present with cognitive impairment or dementia. The most common symptoms that may suggest this condition in the differential diagnosis of rapidly progressive dementia involve retrograde and anterograde amnesia. The diagnosis of this condition is relatively straightforward in patients with established cancer (particularly, small cell lung cancer, germ cell tumors, thymoma) but must also be considered in the setting of occult malignancy as well. Paraneoplastic limbic encephalitis (Fig 3) ${ }^{52}$ is the most classic paraneoplastic disorder with anterograde memory impairment being most prominent; imaging reveals increased signal within the mesial temporal lobes. ${ }^{41}$ FLAIR hyperintensities within the caudate, anterior putamen, and sometimes the medial temporal lobe have been reported in the setting of paraneoplastic syndrome in small cell lung cancer with anti-CV2 antibodies. ${ }^{53}$ Half of those patients with autoimmune dementia diagnosed with CSF autoantibodies will have a paraneoplastic syndrome. Other authors recommend the use of FDG-PET for any patient sus- 

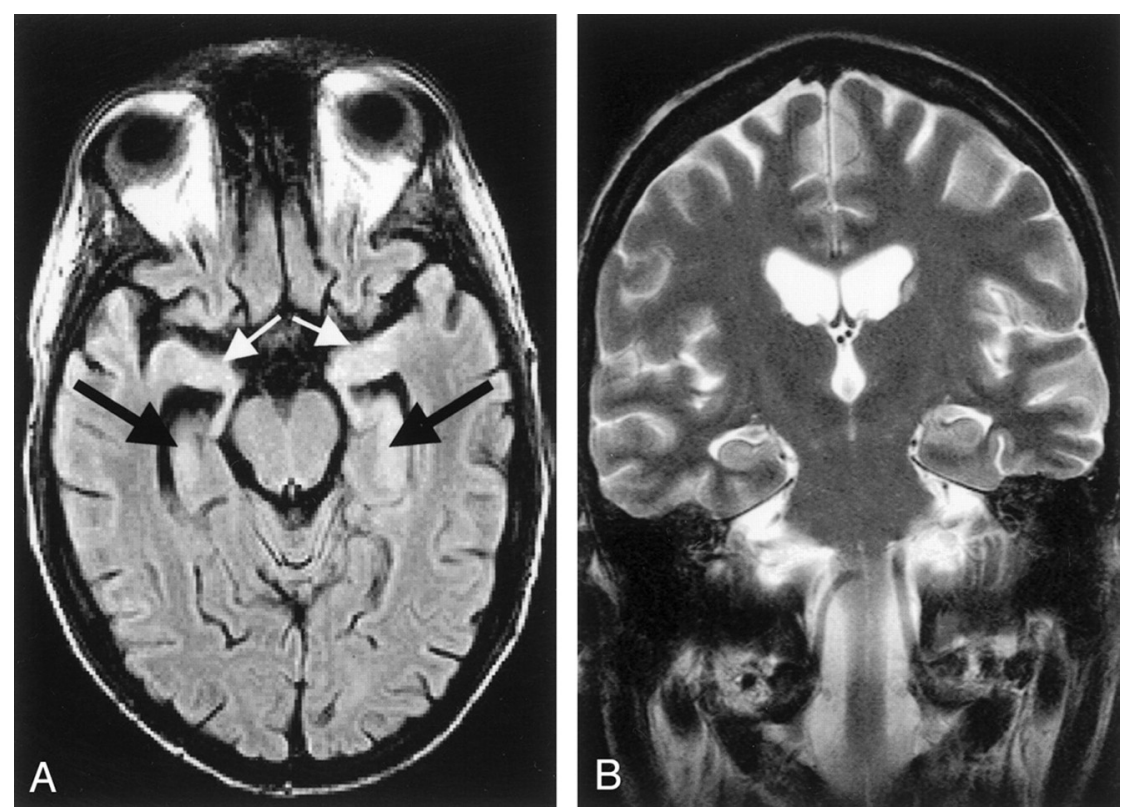

FIG 3. Acute limbic encephalitis. A 26-year-old man with underlying precursor T-cell acute lymphoblastic leukemia developed generalized seizures, had short-term memory loss, and was disoriented in time and place 1 month after undergoing unsuccessful allogeneic bone marrow transplantation. At autopsy, multifocal subacute polioencephalomyelitis in the brain regions that were shown as affected on MR images confirmed the diagnosis of paraneoplastic limbic encephalitis with neuronal loss. Initial MR images were obtained 1 day after the first generalized seizure occurred. A, Axial FLAIR image (TR/TE, 9000/110 ms; TI, $2261 \mathrm{~ms}$ ) shows a slightly elevated signal intensity of both hippocampal formations (black arrows) and amygdala (white arrows). $B$, Coronal conventional T2-weighted turbo spin-echo image (TR/TE/NEX, $4462 \mathrm{~ms} / 120 \mathrm{~ms} / 3$ ) shows no signal-intensity abnormality. ${ }^{52}$

pected of having autoimmune dementia to potentially diagnose underlying malignancy. ${ }^{54}$

Lymphoma. Primary CNS lymphoma is a malignancy of increased recognition and prevalence related to its presence in AIDS. Most of its neurologic manifestations are more related to mass-effect symptoms, but dementia has also been observed, thereby adding this neoplasm to the differential diagnosis of rapid-onset dementias. ${ }^{55}$ Early diagnosis is important for averting the need for extensive resection with poorer outcomes. ${ }^{56} \mathrm{MR}$ imaging categorically reveals solitary or multiple supratentorial mass lesions with minimal edema, generally isointense-to-mildly hyperintense on T1-weighted imaging and hypointense on T2weighted images, and almost always with enhancement. ${ }^{14,57}$ Solitary lesions are more common in immunocompetent individuals. ${ }^{14}$ Of importance, the lesion is capable of crossing the corpus callosum, forming a "butterfly" lesion. ${ }^{57}$ There may also be diffuse extension of the white matter signal change classified as "lymphomatosis cerebri." This form of diffusely infiltrating CNS lymphoma would be more likely to present as a progressive dementia. $^{3}$

The clinical diagnosis of CNS lymphoma is frequently problematic, with nondiagnostic studies leading ultimately to the necessity of brain biopsy for definitive diagnosis; imaging may provide ancillary support to clinical evidence. ${ }^{3}$ MR spectroscopy may be particularly helpful in identifying a neoplastic pattern of metabolite concentrations evidenced by large choline concentrations with lipid and lactate present. ${ }^{14}$ MR perfusion may clarify diag- nosis in contrast to other tumors in that primary lymphoma in the brain has lower relative cerebral blood volume. ${ }^{56}$ Likewise, DWI with ADC maps demonstrate low ADC values compared with glioblastoma and metastatic lesions. ${ }^{56}$ Brain thallium-201 SPECT may be combined with toxoplasma serology to improve diagnostic accuracy in excluding this potential infectious etiology with focal CNS mass lesions. ${ }^{58}$

Intravascular lymphoma, a rare nonHodgkin lymphoma, may likewise generate neurologic symptoms, including subacute dementia, concomitantly with cerebrovascular events. ${ }^{2}$ As a result of its intravascular source, CNS involvement is characterized by diffuse T2-weighted hyperintensity with patchy enhancement and, on occasion, edema., ${ }^{2,59}$ There are some diffusion changes consistent with ischemia in the setting of intravascular lymphoma. ${ }^{60}$ Angiography in these patients does not aid in the diagnosis because intravascular lymphoma appears consistent with vasculitis as a result of the intravascular malignancy. ${ }^{3}$ An antemortem diagnosis of intravascular lymphoma is particularly difficult.

Metastatic CNS Involvement. As with any intracranial process and as the most common cause of brain tumors, metastatic disease may present with rapidly progressive dementia, particularly in the setting of multifocal cortical involvement as has been reported in a few cases of rare miliary cerebral metastases presenting with subacute dementia. ${ }^{61-63}$ Some patients have been reported to develop dementia during treatment as a result of metastatic disease, which could be mistaken for a side effect of therapy (particularly radiation therapy). ${ }^{64}$ In each of these cases, diagnosis of the underlying cause of the dementia is often elusive until postmortem neuropathology. The imaging of most brain lesions consistent with metastatic cancer, aside from miliary spread, is more straightforward, and recognition is important so that patients may benefit from radiation therapy. ${ }^{65}$ Imaging of brain metastases requires a dedicated review of its own. ${ }^{66}$ Briefly, MR imaging generally reveals multiple lesions with a propensity for the graywhite matter junctions with T2 hyperintensity consistent with extensive edema. ${ }^{67}$ Some of these lesions may hemorrhage, as may be evidenced by T1 hyperintensity. DWI and DTI aid in distinguishing metastases from other lesions such as abscesses in the setting of cystic metastasis with a relative lack of restricted diffusion (bright on ADC, dark on DWI); DTI demonstrates displacement of white matter fibers in metastasis. ${ }^{68}$

Lymphomatoid Granulomatosis. Lymphomatoid granulomatosis, a B-cell lymphoma caused by the Epstein-Barr virus with prominent CNS involvement as a result of angiodestructive pathophysiology, is one condition that has been reported to pres- 
ent with dementia in a presenile population. ${ }^{69}$ Generally, pulmonary manifestations exist in almost all patients with CNS manifestations of LG; this unusual constellation may provide the clinical clue necessary to make this diagnosis, whereas those cases with exclusively neurologic involvement are particularly difficult to diagnose. ${ }^{70}$ Multifocal involvement on brain MR imaging is commonplace, with punctate or linear T2 hyperintensities within perivascular spaces with corresponding enhancement on $\mathrm{T} 1$ postcontrast images, particularly within frontal and parietal regions - a finding not exclusive to LG and seen in angiitis and neurosarcoidosis but distinguishable from CNS lymphoma, which most often presents as a solitary lesion. ${ }^{70-73}$ These regions of enhancement correspond on histopathology to granulomatous infiltrates of lymphoid cells and precede hemorrhage. ${ }^{70,72}$ Ringlike enhancement may also appear in the lesions of LG. ${ }^{74}$ Imaging may demonstrate temporal lobe atrophy, perhaps related to lymphocytic infiltration into cerebral blood vessels causing microvascular occlusion and resultant ischemia. ${ }^{69,75}$ Most interesting, research has borne out the importance of MR imaging in this malignancy with evidence that clinical imaging is more sensitive than CSF analysis. ${ }^{73}$

\section{Toxic, Nutritional, and Metabolic Etiologies}

Most nutritional deficiencies that may manifest with neurologic symptoms are slowly progressive. The triad of dermatitis, diarrhea, and dementia, while rarely clinically observed now, characterizes pellagra or niacin deficiency. The role of imaging in this grouping of conditions is much more problematic than in the aforementioned diagnoses because imaging findings, if present, are generally nonspecific and may only offer a glimpse of the correct diagnosis, which may be more readily obtained with thorough history-taking and laboratory studies.

Toxic Causes of Dementia-Like Symptoms. Most cases of neurotoxic exposure are clinically marked by fulminant encephalopathic symptoms of acute onset with a clear source rather than by symptoms of a rapidly progressive dementia. Nevertheless, there are certain imaging features that may support these toxic (generally heavy metal) exposures as the source of neurologic symptoms. T1-weighted hyperintensity within the basal ganglia implies deposition of metals such as iron or manganese. ${ }^{76}$ This finding on MR imaging has been reported in the globus pallidus of individuals exposed to manganese from their occupation as miners who developed cognitive impairment. ${ }^{77}$ The imaging findings in many other exposures are not well-established, and clinical suspicion and appropriate laboratory investigations remain the cornerstone of evaluation of these forms of progressive dementia.

Wernicke Encephalopathy (Thiamine Deficiency). Clinically, the memory loss and cognitive symptoms seen in Wernicke encephalopathy are accompanied by ophthalmoplegias and ataxia; however, as already mentioned, several conditions within the spectrum of rapidly progressive dementia share these symptoms. Moreover, acute Wernicke encephalopathy is underappreciated, particularly in nonalcoholic patients. ${ }^{78}$ Imaging clues may improve recognition of this underdiagnosed clinical entity, with excellent specificity of findings on MR imaging. ${ }^{79}$ There may be T2-weighted and FLAIR hyperintensity within the area around the third ventricle, periaqueductal regions, and bilateral thalami (particularly dorsomedial) in Wernicke encephalopathy-a finding that has been reported even in nonalcoholic cases. ${ }^{78,80,81}$ Moreover, serial imaging illustrates remission of these changes with successful treatment. ${ }^{82}$ DWI improves the neuroimaging evaluation and appears more sensitive to the presumed ischemic and cytotoxic pathophysiology of Wernicke encephalopathy in these regions, evidenced by restricted diffusion. ${ }^{83}$ Therefore, clinical imaging demonstrating gadolinium enhancement or DWI abnormalities within the mammillary bodies and periaqueductal regions and thalami can be of clinical utility in clarifying the diagnosis. ${ }^{78,81}$ These imaging findings may conveniently correlate with the presence of hemorrhagic necrosis within these structures. Ancillary imaging with MR spectroscopy may show decreased or normal NAA with the notable presence of lactate. ${ }^{84,85}$

Wilson Disease. Impaired copper transportation in this autosomal recessive disorder may precipitate either liver disease or neurologic dysfunction, which in the absence of liver disease, presents later in early adulthood. For this reason, progressive dementia with or without other neurologic findings may occur in an atypical age group with Wilson disease. Classically seen on MR imaging is the accumulation of copper within the putamen and thalami reflected by $\mathrm{T} 2$-weighted hyperintensity without evidence of restricted diffusion. ${ }^{86} \mathrm{~A}$ few reports have described the presence of high signal intensity in T2-weighted images of the tegmentum and red nuclei to form the "face of the giant panda" and, inferiorly within the pons, to form the "face of the panda cub," with relative hypointensity of the central portion with adjacent hyperintensity within the aqueduct. ${ }^{86-89}$ While these imaging findings may suggest impaired copper transportation as a cause, ultimately laboratory studies of copper and ceruloplasmin facilitate a definitive diagnosis.

Other Conditions. Metabolic diseases such as mitochondrial disease may also present with a characteristically subcortical dementia; there is impairment in attention and rousability early in the disease process that differentiates them from dementias. Prominent potential causes of subacute dementia are mitochondrial myopathy, encephalopathy, lactic acidosis, and strokelike episodes (MELAS). Imaging findings early in this disease may reveal cortical hyperintensities on FLAIR and T2-weighted images in a gyriform pattern. ${ }^{12}$ DWI also depicts these lesions characteristic of MELAS as hyperintense regions of the cortex. ${ }^{90}$

Osmotic myelinosis is another potential cause of rapidly progressive dementia but will generally present more acutely and involve decreased levels of consciousness that exclude it from the rapidly progressive dementias. Central pontine myelinolysis is visible as a trident-shaped increased T2 signal intensity with sparing of the corticospinal tracts. ${ }^{79}$ Extra-pontine myelinolysis, while rarer, may have T2 prolongation and DWI signal increases in the basal ganglia, thalamus, or cerebellum. The involvement of subcortical structures is an uncommon occurrence, but nevertheless 1 has been reported. ${ }^{79,91}$

\section{CONCLUSIONS}

Presenile and rapidly progressive dementias are a diverse collection of conditions ranging from reversible to intransigent and 
rapidly progressive. Distinctions within this heterogeneous group are best accomplished with a careful and thorough clinical and laboratory examination and may be greatly aided by the use of neuroimaging, particularly MR imaging. We discussed, in the first part of this review, the more frequently encountered neurodegenerative causes of dementia, and in this second installment, we conclude our discussion of rapidly progressive dementias with a review of prion, infectious, inflammatory, autoimmune, neoplastic, and other conditions. The need for further investigation in several areas of clinical investigation of all these conditions is particularly important, especially because a large number of these conditions have overlapping imaging and clinical findings that obfuscate accurate diagnosis. Both the clinician and radiologist alike should be open to the possibility of alternate diagnoses in the setting of rapidly progressive presenile dementias, particularly because several diseases within this group benefit from urgent and specific treatment.

\section{REFERENCES}

1. Heinemann U, Gawinecka J, Schmidt C, et al. Differential diagnosis of rapid progressive dementia. Eur Neurol Rev 2010;5:21-28

2. Geschwind MD, Haman A, Miller BL. Rapidly progressive dementia. Neurol Clin 2007;25:783-807, vii

3. Geschwind MD, Shu H, Haman A, et al. Rapidly progressive dementia. Ann Neurol 2008;64:97-108

4. Lee H, Hoffman C, Kingsley PB, et al. Enhanced detection of diffusion reductions in Creutzfeldt-Jakob disease at a higher $B$ factor. AJNR Am J Neuroradiol 2010;31:49-54

5. Hyare H, Thornton J, Stevens J, et al. High-b-value diffusion MR imaging and basal nuclei apparent diffusion coefficient measurements in variant and sporadic Creutzfeldt-Jakob disease. AJNR Am J Neuroradiol 2010;31:521-26

6. Smart JM, Wood A. Value of fluid-attenuated inversion recovery MR imaging in an unusual case of sporadic Creutzfeldt-Jakob disease. AJR Am J Roentgenol 2001;177:948 -49

7. Meissner B, Kallenberg K, Sanchez-Juan P, et al. Isolated cortical signal increase on MR imaging as a frequent lesion pattern in sporadic Creutzfeldt-Jakob disease. AJNR Am J Neuroradiol 2008;29:1519-24

8. Collie DA, Sellar RJ, Zeidler M, et al. MRI of Creutzfeldt-Jakob disease: imaging features and recommended MRI protocol. Clin Radiol 2001;56:726-39

9. Vitali P, Maccagnano E, Caverzasi E, et al. Diffusion-weighted MRI hyperintensity patterns differentiate CJD from other rapid dementias. Neurology 2011;76:1711-19

10. Zerr I, Kallenberg K, Summers DM, et al. Updated clinical diagnostic criteria for sporadic Creutzfeldt-Jakob disease. Brain 2009;132: 2659-68

11. Almeida OP, Lautenschlager NT. Dementia associated with infectious diseases. Int Psychogeriatr 2005;17(suppl 1):S65-77

12. Ukisu R, Kushihashi T, Tanaka E, et al. Diffusion-weighted MR imaging of early-stage Creutzfeldt-Jakob disease: typical and atypical manifestations. Radiographics 2006;26(suppl 1):S191-204

13. Misra U, Kalita J, Phadke R, et al. Usefulness of various MRI sequences in the diagnosis of viral encephalitis. Acta Trop 2010;116:206-11

14. Küker W, Nagele T, Korfel A, et al. Primary central nervous system lymphomas (PCNSL): MRI features at presentation in 100 patients. J Neurooncol 2005;72:169-77

15. McCabe K, Tyler K, Tanabe J Diffusion-weighted MRI abnormalities as a clue to the diagnosis of herpes simplex encephalitis. Neurology 2003;61:1015-16

16. Sawlani V. Diffusion-weighted imaging and apparent diffusion coefficient evaluation of herpes simplex encephalitis and Japanese encephalitis. J Neurol Sci 2009;287:221-26
17. Prakash M, Kumar S, Gupta RK. Diffusion-weighted MR imaging in Japanese encephalitis. J Comput Assist Tomogr 2004;28:756-61

18. Satishchandra P, Sinha S. Relevance of neuroimaging in the diagnosis and management of tropical neurologic disorders. Neuroimaging Clin N Am 2011;21:737-56, vii

19. Tucker KA, Robertson KR, Lin W, et al. Neuroimaging in human immunodeficiency virus infection. J Neuroimmunol 2004;157: $153-62$

20. Ragin AB, Storey P, Cohen BA, et al. Disease burden in HIV-associated cognitive impairment: a study of whole-brain imaging measures. Neurology 2004;63:2293-97

21. Ragin AB, Storey $\mathrm{P}$, Cohen $\mathrm{BA}$, et al. Whole brain diffusion tensor imaging in HIV-associated cognitive impairment. AJNR Am J Neuroradiol 2004;25:195-200

22. Ernst $\mathrm{T}$, Jiang CS, Nakama $\mathrm{H}$, et al. Lower brain glutamate is associated with cognitive deficits in HIV patients: a new mechanism for HIV-associated neurocognitive disorder. J Magn Reson Imaging 2010;32:1045-53

23. Waniek C, Prohovnik I, Kaufman AA, et al. Rapidly progressive frontal-type dementia associated with Lyme disease. J Neuropsychiatry Clin Neurosci 1995;7:345-47

24. Hildenbrand P, Craven D, Jones R, et al. Lyme neuroborreliosis: manifestations of a rapidly emerging zoonosis. AJNR Am J Neuroradiol 2009;30:1079-87

25. Massengo SA, Bonnet F, Braun C, et al. Severe neuroborreliosis: the benefit of prolonged high-dose combination of antimicrobial agents with steroids-an illustrative case. Diagn Microbiol Infect Dis 2005;51:127-30

26. Fernandez RE, Rothberg M, Ferencz G, et al. Lyme disease of the CNS: MR imaging findings in 14 cases. AJNR Am J Neuroradiol 1990; 11:479-81

27. Agarwal R, Sze G. Neuro-lyme disease: MR imaging findings. Radiology 2009;253:167-73

28. Agosta F, Rocca MA, Benedetti B, et al. MR imaging assessment of brain and cervical cord damage in patients with neuroborreliosis. AJNR Am J Neuroradiol 2006;27:892-94

29. Falcone S, Quencer RM, Post MJ. Magnetic resonance imaging of unusual intracranial infections. Top Magn Reson Imaging 1994;6:41-52

30. Hoffmann M, Muniz J, Carroll E, et al. Cryptococcal meningitis misdiagnosed as Alzheimer's disease: complete neurological and cognitive recovery with treatment. J Alzheimers Dis 2009;16:517-20

31. Oztürk A, Gurses C, Baykan B, et al. Subacute sclerosing panencephalitis: clinical and magnetic resonance imaging evaluation of 36 patients. J Child Neurol 2002;17:25-29

32. Sener RN. Subacute sclerosing panencephalitis findings at MR imaging, diffusion MR imaging, and proton MR spectroscopy. AJNR Am J Neuroradiol 2004;25:892-94

33. Tuncay R, Akman-Demir G, Gokyigit A, et al. MRI in subacute sclerosing panencephalitis. Neuroradiology 1996;38:636-40

34. Alkan A, Sarac K, Kutlu R, et al. Early- and late-state subacute sclerosing panencephalitis: chemical shift imaging and single-voxel MR spectroscopy. AJNR Am J Neuroradiol 2003;24:501-06

35. Trivedi R, Gupta RK, Agarawal A, et al. Assessment of white matter damage in subacute sclerosing panencephalitis using quantitative diffusion tensor MR imaging. AJNR Am J Neuroradiol 2006;27:1712-16

36. Kelley BJ, Boeve BF, Josephs KA. Rapidly progressive young-onset dementia. Cogn Behav Neurol 2009;22:22-27

37. Longley WA. Multiple sclerosis-related dementia: relatively rare and often misunderstood. Brain Impairment 2007;8:154-67

38. Pelletier D, Garrison K, Henry R. Measurement of whole-brain atrophy in multiple sclerosis. J Neuroimaging 2004;14:11S-19S

39. Anderson VM, Fox NC, Miller DH. Magnetic resonance imaging measures of brain atrophy in multiple sclerosis. J Magn Reson Imaging 2006;23:605-18

40. Castillo P, Woodruff B, Caselli R, et al. Steroid-responsive encephalopathy associated with autoimmune thyroiditis. Arch Neurol 2006; 63:197-202 
41. Vernino S, Geschwind M, Boeve B. Autoimmune encephalopathies. Neurologist 2007;13:140-47

42. Pickuth D, Spielmann R, Heywang-Kobrunner S. Role of radiology in the diagnosis of neurosarcoidosis. Eur Radiol 2000;10:941-44

43. Christoforidis GA, Spickler EM, Recio MV, et al. MR of CNS sarcoidosis: correlation of imaging features to clinical symptoms and response to treatment. AJNR Am J Neuroradiol 1999;20:655-69

44. Lury KM, Smith JK, Matheus MG, et al. Neurosarcoidosis: review of imaging findings. Semin Roentgenol 2004;39:495-504

45. Giannini C, Salvarani C, Hunder G, et al. Primary central nervous system vasculitis: pathology and mechanisms. Acta Neuropathol 2012;123:759-72

46. Salvarani C, Brown RD Jr, Calamia KT, et al. Primary central nervous system vasculitis: analysis of 101 patients. Ann Neurol 2007;62:442-51

47. Zelnik N, Pacht A, Obeid R, et al. Range of neurologic disorders in patients with celiac disease. Pediatrics 2004;113:1672-76

48. Pengiran Tengah DS, Wills AJ, Holmes GK. Neurological complications of coeliac disease. Postgrad Med J 2002;78:393-98

49. Cicarelli G, Della Rocca G, Amboni M, et al. Clinical and neurological abnormalities in adult celiac disease. Neurol Sci 2003;24:311-17

50. Kieslich M, Errazuriz G, Posselt HG, et al. Brain white-matter lesions in celiac disease: a prospective study of 75 diet-treated patients. $P e$ diatrics 2001;108:e21

51. Addolorato G, Di Giuda D, De Rossi G, et al. Regional cerebral hypoperfusion in patients with celiac disease. Am J Med 2004;116:312-17

52. Thuerl C, Muller K, Laubenberger J, et al. MR imaging of autopsyproved paraneoplastic limbic encephalitis in non-Hodgkin lymphoma. AJNR Am J Neuroradiol 2003;24:507-11

53. Vernino $\mathrm{S}$, Tuite $\mathrm{P}$, Adler $\mathrm{CH}$, et al. Paraneoplastic chorea associated with CRMP-5 neuronal antibody and lung carcinoma. Annal Neurol 2002;51:625-30

54. Murray AD. Imaging approaches for dementia. AJNR Am J Neuroradiol 2011;33:1836-44

55. Bataille B, Delwail V, Menet E, et al. Primary intracerebral malignant lymphoma: report of 248 cases. J Neurosurg 2000;92:261-66

56. Hussien AR, Mangla R, Liu X, et al. An approach to improve the diagnosis of primary CNS lymphoma using conventional and advanced imaging techniques. Contemporary Diagn Radiol 2012;35:1-8

57. Slone HW, Blake JJ, Shah R, et al. CT and MRI findings of intracranial lymphoma. AJR Am J Roentgenol 2005;184:1679-85

58. Skiest DJ, Erdman W, Chang WE, et al. SPECT thallium-201 combined with toxoplasma serology for the presumptive diagnosis of focal central nervous system mass lesions in patients with AIDS. J Infect 2000;40:274-81

59. Vieren $M$, Sciot R, Robberecht W. Intravascular lymphomatosis of the brain: a diagnostic problem. Clin Neurol Neurosurg 1999;101:33-36

60. Baehring JM, Henchcliffe C, Ledezma CJ, et al. Intravascular lymphoma: magnetic resonance imaging correlates of disease dynamics within the central nervous system. J Neurol Neurosurg Psychiatry 2005;76:540-44

61. Bouza G, Roel JE, Galich M, et al. Rapidly progressive dementia caused by tumor microembolism in the central nervous system [in Spanish]. Medicina (B Aires) 1997;57:447-50

62. Fontan A, Zarranz J, Camarero C, et al. Dementia caused by miliary cerebral metastasis of a hepatocarcinoma [in Spanish]. Med Clin (Barc) 1989;93:421-23

63. Rivas E, Sanchez-Herrero J, Alonso M, et al. Miliary brain metastases presenting as rapidly progressive dementia. Neuropathology 2005; 25:153-58

64. Yamao Mochizuki S, Nishimura N, Inoue A, et al. Miliary brain metastases in 2 cases with advanced non-small cell lung cancer harboring EGFR mutation during gefitinib treatment. Respir Investig 2012; 50:117-21

65. Tosoni A, Ermani M, Brandes AA. The pathogenesis and treatment of brain metastases: a comprehensive review. Crit Rev Oncol Hematol 2004;52:199-215

66. Barajas RF Jr, Cha S. Imaging diagnosis of brain metastasis. Prog Neurol Surg 2012;25:55-73
67. Grainger RG, Allison DJ, Adam A, et al. Diagnostic Radiology: A Textbook of Medical Imaging. London: Churchill Livingstone; 2001

68. Tsuchiya K, Fujikawa A, Nakajima M, et al. Differentiation between solitary brain metastasis and high-grade glioma by diffusion tensor imaging. Br J Radiol 2005;78:533-37

69. Carone DA, Benedict RHB, Zivadinov R, et al. Progressive cerebral disease in lymphomatoid granulomatosis causes anterograde amnesia and neuropsychiatric disorder. J Neuroimaging 2006;16:163-66

70. Tateishi U, Terae S, Ogata A, et al. MR imaging of the brain in lymphomatoid granulomatosis. AJNR Am J Neuroradiol 2001;22:1283-90

71. Kawai N, Miyake K, Nishiyama Y, et al. FDG-PET findings of the brain in lymphomatoid granulomatosis. Ann Nucl Med 2006;20:683-87

72. Miura H, Shimamura H, Tsuchiya K, et al. Magnetic resonance imaging of lymphomatoid granulomatosis: punctate and linear enhancement preceding hemorrhage. Eur Radiol 2003;13:2192-95

73. Patsalides AD, Atac G, Hedge U, et al. Lymphomatoid granulomatosis: abnormalities of the brain at MR imaging. Radiology 2005; 237:265-73

74. Kiryu S, Okubo T, Takeuchi K, et al. Magnetic resonance imaging and diffusion tensor analysis of lymphomatoid granulomatosis of the brain. Acta Radiol 2006;47:509-13

75. Mizuno T, Takanashi $\mathrm{Y}$, Onodera $\mathrm{H}$, et al. A case of lymphomatoid granulomatosis/angiocentric immunoproliferative lesion with long clinical course and diffuse brain involvement. J Neurol Sci 2003;213:67-76

76. Yoshikawa K, Matsumoto M, Hamanaka M, et al. A case of manganese induced parkinsonism in hereditary haemorrhagic telangiectasia. J Neurol Neurosurg Psychiatry 2003;74:1312-14

77. Josephs K, Ahlskog J, Klos K, et al. Neurologic manifestations in welders with pallidal MRI T1 hyperintensity. Neurology 2005;64: 2033-39

78. Unlu E, Cakir B, Asil T. MRI findings of Wernicke encephalopathy revisited due to hunger strike. Eur J Radiol 2006;57:43-53

79. Lim CC. Neuroimaging in postinfectious demyelination and nutritional disorders of the central nervous system. Neuroimaging Clin $N$ Am 2011;21:843-58, viii

80. White ML, Zhang Y, Andrew LG, et al. MR imaging with diffusionweighted imaging in acute and chronic Wernicke encephalopathy. AJNR Am J Neuroradiol 2005;26:2306-10

81. Fei GQ, Zhong C, Jin L, et al. Clinical characteristics and MR imaging features of nonalcoholic Wernicke encephalopathy. AJNR Am J Neuroradiol 2008;29:164-69

82. Zhong C, Jin L, Fei G. MR imaging of nonalcoholic Wernicke encephalopathy: a follow-up study. AJNR Am J Neuroradiol 2005;26: 2301-05

83. Halavaara J, Brander A, Lyytinen J, et al. Wernicke's encephalopathy: is diffusion-weighted MRI useful? Neuroradiology 2003;45:519-23

84. Rugilo CA, Uribe Roca MC, Zurru MC, et al. Proton MR spectroscopy in Wernicke encephalopathy. AJNR Am J Neuroradiol 2003;24: 952-55

85. Murata T, Fujito T, Kimura H, et al. Serial MRI and (1)H-MRS of Wernicke's encephalopathy: report of a case with remarkable cerebellar lesions on MRI. Psychiatry Res 2001;108:49-55

86. Singh P, Ahluwalia A, Saggar K, et al. Wilson's disease: MRI features. J Pediatr Neurosci 2011;6:27-28

87. Mascalchi M, Vella A, Ceravolo R. Movement disorders: role of imaging in diagnosis. J Magn Reson Imaging 2012;35:239-56

88. Shivakumar R, Thomas SV. Teaching NeuroImages: face of the giant panda and her cub-MRI correlates of Wilson disease. Neurology 2009;72:E50

89. Jacobs DA, Markowitz CE, Liebeskind DS, et al. The "double panda sign" in Wilson's disease. Neurology 2003;61:969-69

90. Wang XY, Noguchi K, Takashima S, et al. Serial diffusion-weighted imaging in a patient with MELAS and presumed cytotoxic oedema. Neuroradiology 2003;45:640-43

91. Tatewaki $Y$, Kato K, Tanabe $Y$, et al. MRI findings of corticosubcortical lesions in osmotic myelinolysis: report of two cases. Br J Radiol 2012;85:e87-90 